\title{
Sea saline by vyana scientific labs, a revolution in the herbal natural mouth rinse race
}

\section{Editorial}

Over years of dental practice, doctors (we) always advise patients for warm saline gargles especially after surgical procedures. It ranges from a procedure as small as an oral prophylaxis to as big as a surgery involving the teeth. The thought must have crossed our minds, that we have never specified the type of water that needs to be used or the amount of salt that is to be added. The water quality and its constituents is a cause of growing concern in our country. We recently came across this very interesting yet important concept of Sea Saline by Vyana Scientific Labs which is an alcohol free mouthwash enhanced with salt and refreshing clove to promote healing post procedures. The key ingredients are distilled water, Salt and Clove oil.

\section{Salt in the history of medicine}

Salt has been used as a medicine and a disinfecting agent all through the history. In $450 \mathrm{BC}$ both sea salt and rock salt were used in the healing methods by Hippocrates. Various salt based potions and ointments were used for ulcers, skin diseases and respiratory problems. Roman doctors dispensed drinks and ointments made with salt and during the Renaissance period doctors recommended salt baths for skin diseases and itching. This ancient use of salt continues all the way to the 20th century and has growing popularity amongst naturalists. We are now well aware of the medicinal properties of salt and its advantages in treating diseases. Today, salt is an integral to popular natural healing mechanism used in the form of inhalations, salt-water baths and drinking-therapy etc. An important discovery of 20th century medicine is that salt water - in the form of an isotonic sodium chloride (saline) solution - has the same fluid quality as blood plasma.

\section{How does salt work as an oral rinsing agent}

The mouthwash reduces the bacterial load in dental plaque by creating an alkaline medium temporarily (increases the $\mathrm{pH}$ of the saliva) creating an environment in hospitable for bacteria. An acidic environment is ideal for the growth and multiplication of bacteria. Being an isotonic solution it does no harm to the soft tissue in the oral cavity. Another major advantage is that the mouthwash is no alcohol based, thus it causes no burning sensation or harm to the tissues (hard and soft tissues) which are mainly seen in almost all mouth washes available in the market Alcohol based compositions is a major reason we have never promoted off the shelf mouthwashes.

\section{Distilled water}

The common home water used for salt water gargles is usually contaminated with organism growth which remains active post filtration or is adulterated. Thus leading to increased bacterial load in the mouth potentially dangerous especially after surgical procedures. Distilled water is water that has had many of its impurities removed through the process of distillation. Use of distilled water as a mouthwash ingredient reduces contamination by a huge percentage, creating a healthy environment in patient's mouth post surgical

\author{
Volume 5 Issue 6 - 2016
}

\author{
Saurabh Lall \\ Department of Periodontology and Implant Dentistry, ESIC \\ Dental College \& Hospital, India
}

Correspondence: Department of Periodontology and Implant Dentistry, ESIC Dental College \& Hospital, India, Emailsaurabhlall.delhi@gmail.com

Received: December 0I, 2016 | Published: December 2I, 2016

procedures. All of this is beneficial in the healing process as addition of contamination in the use of tap water can interfere with the healing process.

\section{Clove}

For centuries clove has been known to relieve all of us from tooth pain. Its established use spans across Ayurvedic medicine, Chinese medicine and in dentistry where it acts as a natural analgesic and anesthetic in dental emergencies. The aromatic oil received from clove provides a warming effect in the mouth which is very soothing. Clove is instrumental in decreasing post-operative pain. Considered by many as a wonder drug, Clove Oil is known to have Anti Bacterial, Anti Oxidant, Anti Inflammatory and Anti Fungal properties, thus making it the magic component of Sea Saline. Clove oil is expected to act as an Immunity Booster, Pain Reliever and boost for general well being and oral hygiene.

\section{Over all benefits of Saline mouthrinses over regular mouth washes}
a. Natural product
b. Non-alcohol based
c. Boost General Hygiene
d. Immunity Booster
e. Isotonic solution
f. Bacteria free product
g. Multiple usages (post dental procedures, mouth ulcers)
h. Analgesic and warming effect of clove .

\section{Precaution}

People suffering from hypertension should be careful while use of such rinses.

\section{Acknowledgments}

None. 


\section{Conflicts of interest}

The authors declare that there are no conflicts of interest.

\section{Funding details}

None. 